\title{
Next-Generation \\ Mobile Satellite Networks
}

Enrico Del Re and Laura Pierucci, University of Florence

\begin{abstract}
Due to the increasing demands of multimedia services supporting high bit rates and mobility, ATM, TCP/IP, and satellite technology are going to be associated to form the internetwork infrastructure of future global systems. In this scenario, distinctions between terrestrial and satellite communications systems, as well as between fixed networks and $3 \mathrm{G}$ mobile networks, will cease to exist in a global coverage wireless system. The European Action COST252 actively participated in developing the satellite component of the UMTS system.
\end{abstract}

\section{INTRODUCTION}

A new digital society, globally interconnected, with multimedia applications, information on demand, and delivery of advanced data services at low cost is the user's expectation and the operator's promise.

The objective of the International Mobile Telecommunications after the year 2000/Universal Mobile Telecommunications System (IMT2000/UMTS) is to extend the services provided by current second-generation $(2 \mathrm{G})$ systems with high data rate capabilities to support a wireless Internet access, as well as simultaneous voice and data, email, and broadband integrated services.

The satellite component of UMTS (S-UMTS) will provide global coverage and roaming of UMTS users in developing countries, thus maintaining terminal compatibility and service flexibility. It is internationally recognized that satellite systems are needed to provide the required global coverage of future fixed and mobile communications. The satellite segment will play a significant role within the global information infrastructure (GII).

The European Action COST252, "Evolution of Satellite Personal Communication from Second to Future Generation Systems," promoted a coordinated effort of research teams in 11 European countries toward the achievement of future satellite-terrestrial systems by studying a smooth migration from $2 \mathrm{G}$ terrestrial systems (e.g.,
GSM, DCS 1800, DECT) to include the satellite component. The COST252 activities can be summarized as follows:

- A mid-term activity focused on the adaptability of the Global System for Mobile Communications (GSM) standard to mobile satellite systems (MSS) by considering the future requirements of an integrated thirdgeneration ( $3 \mathrm{G})$ system (UMTS).

- A longer-term activity dealing with the exploitation of the satellite component for mobile broadband services (MBS), capable of providing services at higher rates $(>2 \mathrm{Mb} / \mathrm{s})$ than presently defined in the UMTS.

In Europe, the $3 \mathrm{G}$ mobile system is aimed at delivering advanced multimedia services at data rates of $144 \mathrm{~kb} / \mathrm{s}$ (preferably $384 \mathrm{~kb} / \mathrm{s}$ ) for highmobility users with wide coverage, and of $2 \mathrm{Mb} / \mathrm{s}$ for low-mobility users with local coverage on the terrestrial segment and limited to $144 \mathrm{~kb} / \mathrm{s}$ on the satellite connection. On the other hand, under Advanced Communications Technologies and Services (ACTS) projects and Ka band commercial systems (SPACEWAY, ASTROLINK, EUROSKYWAY, TELEDESIC), the focus of the satellite segment points to higher-data-rate multimedia services mainly directed to fixed and possibly portable terminals.

The European Space Agency (ESA) is actively participating in the standardization of the $3 \mathrm{G}$ global multisatellite multibeam systems with autonomous proposals based on the wideband code-division access technique, satellite wideband code-division multiple access (SWCDMA), and hybrid satellite wideband code/time-division multiple access (SWCTDMA), which combines CDMA with the known TDMA advantages.

This article addresses research and results of COST252 dealing with the satellite part of UMTS: the satellite constellation and scenarios, networking infrastructure requirements, air interface aspects, CDMA receivers for a global coverage wireless system with different services at variable bit rates, optimum resource management , and guaranteed quality of service (QoS). 


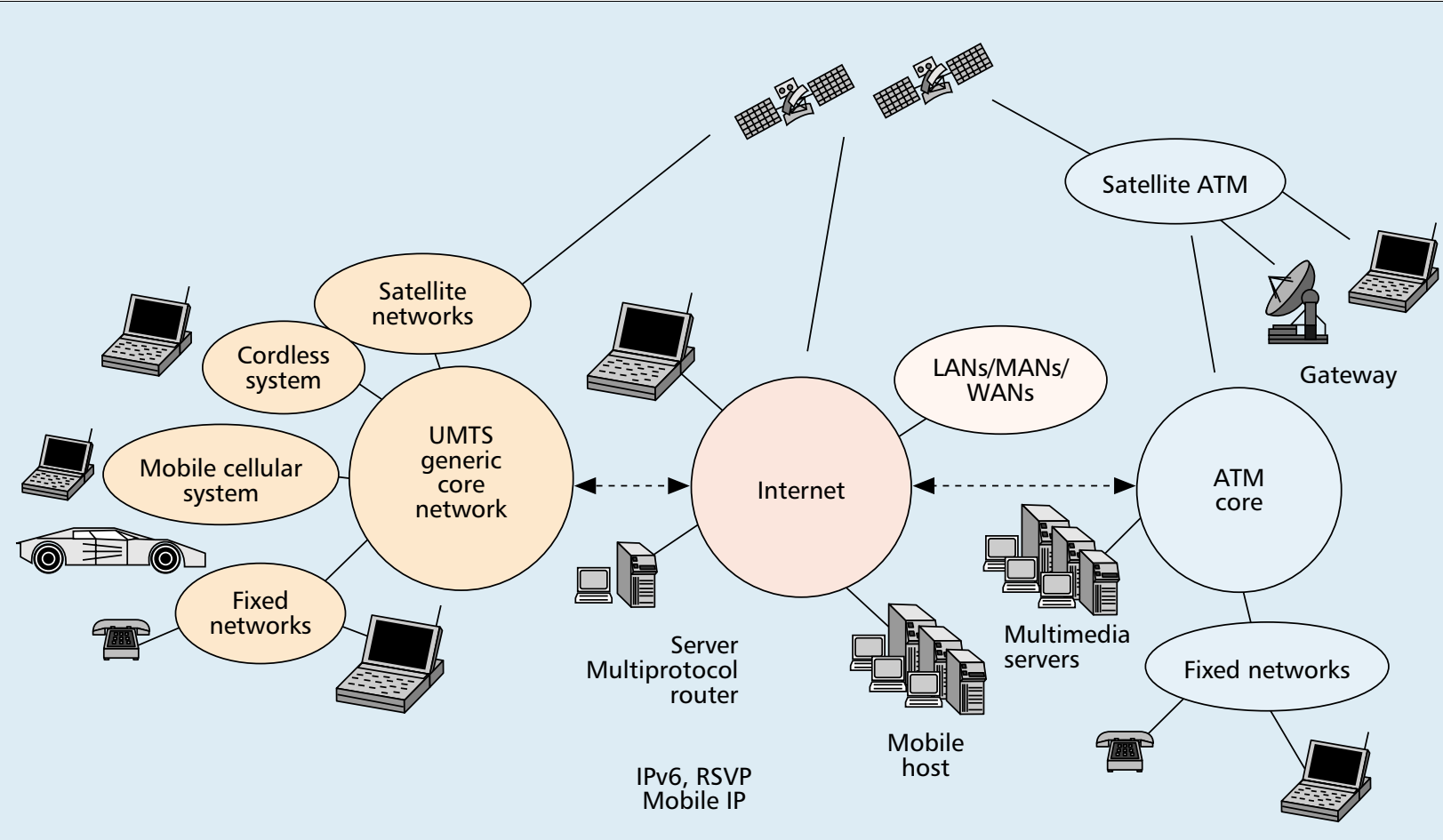

IP over ATM over satellite

Figure 1. Multimedia services in the future global system.

\section{SATELLITE System ARCHITECTURE}

\section{SYSTEM SCENARIOS}

Satellite connections can help the exponential growth of the Internet with applications based on TCP/IP, especially when multimedia services require high bandwidth or bandwidth-on-demand flexibility. Therefore, asynchronous transfer mode (ATM), TCP/IP, and satellite technology are going to form the internetwork infrastructure of future global systems.

In considering the satellite as part of the network infrastructure, it is necessary to analyze the interoperability of this system with the terrestrial backbone networks in order to guarantee the same QoS characteristics and compatibility of different types of services.

The following scenarios $[1,2]$ were considered in the analysis of future satellite (S) services as shown in Fig. 1.

The S-UMTS Scenario - This scenario requires the S-UMTS network to be interfaced with the mobile terrestrial network to provide high-rate services up to $2 \mathrm{Mb} / \mathrm{s}$.

The S-ATM Scenario - Transmission of ATMlike cells via satellite by assuming a broadband integrated services digital network (B-ISDN) backbone network. The aim is to bring the ATM functionality closer to the user terminal without violating its connection-oriented features and the guaranteed QoS.

The S-IP Scenario - Internet protocol transmissions allowing direct connection to an IP backbone network with the adoption of the new
Internet standards such as IPv6, Resource Reservation Protocol (RSVP), and Mobile IP. Satellite systems with direct intersatellite links (ISLs) can use redundant paths to avoid network congestion, while IP routing schemes provide true challenges in an end-to-end packet network. IP routing can be attractive in low Earth orbit (LEO) satellite meshed networks, which are poorly suited to circuit switching, as well as to support multicasting and simplified internetworking with many ground IP-based networks. However, other commercial systems have adopted a different approach: Celestri and SkyBridge incorporate ATM variants for satellite switching, and Teledesic proposes fast packet switching using proprietary connectionless adaptive routing protocols.

\section{SYSTEM REQUIREMENTS}

Capacity - In UMTS the satellite component, S-UMTS, is used to provide mobile multimedia services to individual users at a rate up to 144 $\mathrm{kb} / \mathrm{s}$. Broadband satellite systems in $\mathrm{Ka}$ band with onboard processing and ISLs will provide to each customer a high data rate for fixed-type terminals in the near future: Teledesic will allow $16 \mathrm{~kb} / \mathrm{s}-2 \mathrm{Mb} / \mathrm{s}$ uplink and $16 \mathrm{~kb} / \mathrm{s}-64$ $\mathrm{Mb} / \mathrm{s}$ downlink; Spaceway $16 \mathrm{~kb} / \mathrm{s}-6 \mathrm{Mb} / \mathrm{s}$ uplink and up to $92 \mathrm{Mb} / \mathrm{s}$ downlink; and Astrolink up to $20 \mathrm{Mb} / \mathrm{s}$ in uplink and up to $155 \mathrm{Mb} / \mathrm{s}$ in downlink.

Frequency - At present, the global standard for UMTS is in the frequency bands 1885-2025 $\mathrm{MHz}$ and 2110-2200 MHz with only a subband of $30 \mathrm{MHz}$ reserved to the satellite component. Traditionally, MSS operate in L and S bands for uplink and downlink, respectively, while feeder 


\begin{tabular}{lllllllllll} 
& \multicolumn{4}{c}{ Constellation } & & Sat. altitude & $\varepsilon_{\min }$ & Orbit period & Satellite speed & Max. range \\
\hline & $N$ & $P$ & $Q$ & $m$ & $h[\mathrm{~km}]$ & {$\left[{ }^{\circ}\right]$} & $T[\mathrm{~s}]$ & $v_{s}[\mathrm{~km} / \mathrm{s}]$ & $\psi_{\max }\left[{ }^{\circ}\right]$ \\
\hline Inclined MEO & 27 & 9 & 3 & $5 / 3$ & 10,354 & 40 & 21,541 & 4.881 & 33.02 \\
\hline Polar MEO & 28 & 4 & 7 & $N / A$ & 10,354 & 40 & 21,541 & 4.881 & 33.02 \\
\hline Polar LEO & 96 & 8 & 12 & N/A & 1666 & 30 & 7180 & 7.039 & 16.63
\end{tabular}

Table 1. Orbital parameters: total number of satellites $\mathrm{N}$, number of orbit planes $\mathrm{P}$, number of satellites in each orbit plane $\mathrm{Q}$, and harmonic factor, $\mathrm{m}$, which denotes initial distribution of satellites over the sphere.

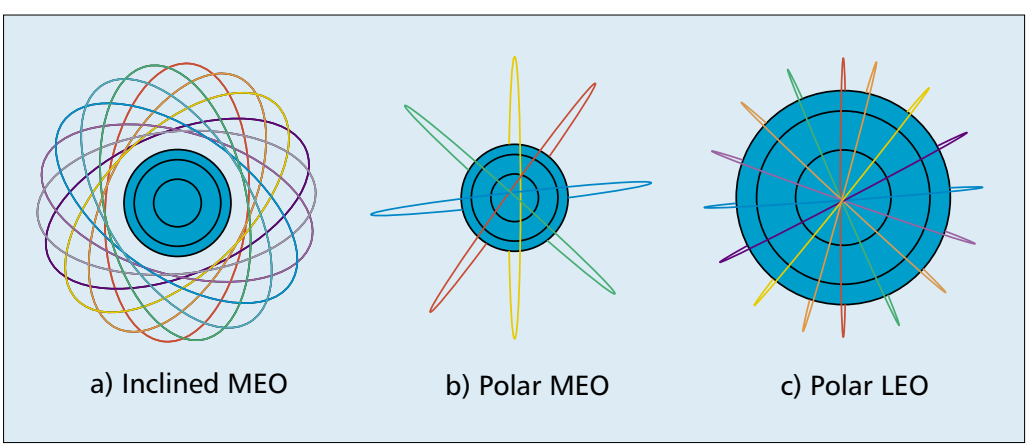

Figure 2. A polar view at the selected constellation.

links have been established in the $\mathrm{C}$ band for conventional narrowband services, but these will have to use higher frequencies in the $\mathrm{Ka}(20-30$ $\mathrm{GHz})$ and extra high frequency (EHF) (40-50 $\mathrm{GHz}$ ) bands in order to offer broadband services to mobile users.

\section{Satellite Constellation}

Currently, the vast majority of existing satellite systems are geostationary Earth orbit (GEO) without onboard switching or intersatellite routing. GEO performance is affected by the inherent delay (round-trip rime of $0.5 \mathrm{~s}$ ) due to the distance of the satellite from the Earth's surface, which is undesirable for real-time traffic as well as the probability of bit errors on satellite links. This type of orbit generally offers poor visibility to a mobile terminal in suburban and urban areas.

In new broadband systems with shorter propagation delays, the satellites must be available at high elevation angles; therefore, a high number of satellites is required in nongeostationary constellations, medium Earth orbit (MEO) and low Earth Orbit (LEO).

LEO satellites (at altitude elevation ranging 500-2000 km above the Earth's surface) provide low round-trip delay $(10-40 \mathrm{~ms})$ compared to 250 ms of GEO, but the orbital height of a LEO limits the size of the coverage spot (footprints), and a high number of satellites is needed. The high satellite speed with respect to the ground means that the transmission will be subject to significant Doppler variation. In order to maintain continuous real-time transmission, intersatellite handovers are frequently required, and the use of multiple spotbeam arrays means that an interspotbeam handover will be a huge signaling task for these constellations.

MEO networks (distance from Earth's sur- face $3000 \mathrm{~km}-\mathrm{GEO}$ orbit) seem to be a good compromise between the high orbital distances of the GEO (with no extra complexity for handoff services) and LEO distances (with very high handover rates). In addition, each satellite can be viewed for over $1 \mathrm{~h}$ before the user switches to the next satellite.

Other constellations are available. For example, high elliptic orbit (HEO) satellites with a large difference between the perigee and apogee, such as the commercial Ellipso and Pentriad, only offer communication services when the satellites move slowly around the apogee. Nevertheless, the development of these systems remains confined to specific and local services.

By taking into account different orbital parameters and constellation characteristics, three satellite constellations have been selected (Table 1) for trade-off simulation results [2, 3] of satellite visibility parameters, a single satellite coverage area, and intersatellite and interspotbeam handover time.

Polar views at the three selected constellations are shown in Fig. 2; Fig. 3 shows coverage areas of satellite footprints on the Earth [16].

\section{Network ArChitecture}

The choice of satellite constellation has a great impact on a broadband satellite system design. As far as the satellite ground network is concerned, the number and location of fixed Earth stations (FES) depend on the constellation parameters and provision of ISLs, as well as on the feeder link bandwidth requirements.

Another important parameter is the dependence on the terrestrial network infrastructure. The satellite links are essential for interstation signaling when there is no terrestrial infrastructure deployed. For example, most GEO systems do not need ground station interconnections through terrestrial links, whereas non-GEO systems require only a few satellite links to the FESs, when ISLs are used; otherwise, they highly depend on a fast backbone network. In GEO systems, ISLs mainly handle the traffic between different regions of the Earth and bypass the terrestrial links, whereas in most non-GEO constellations ISLs are essential in order to reduce the number of FESs.

Figure 4 shows a dynamic multispotbeam satellite network architecture and its interconnection to a fixed core network [4]. Each satellite coverage area is serviced by at least one Earth station that is connected to the fixed network and plays the role of the satellite network gateway (GTW). ISLs are used to minimize the 
(a)

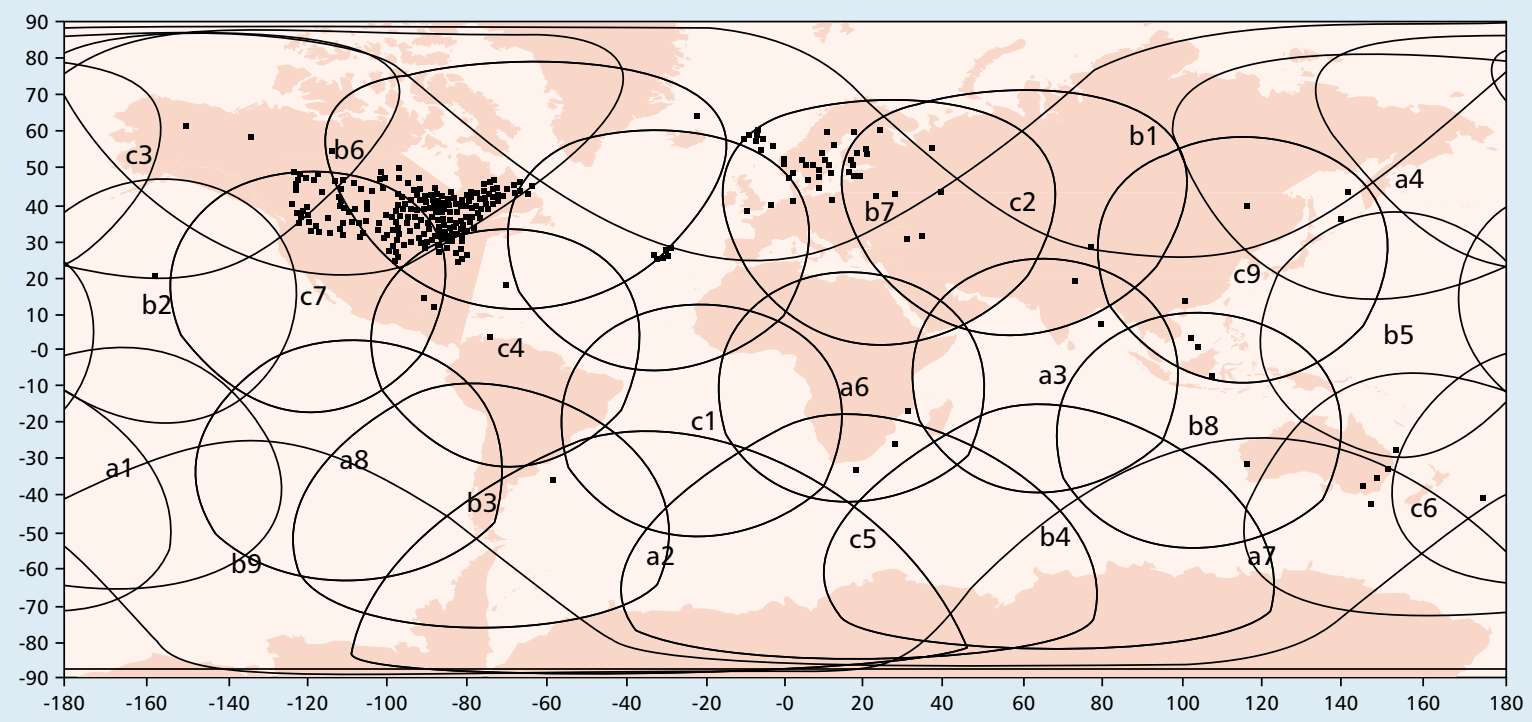

Elevation constraint $=40.0$

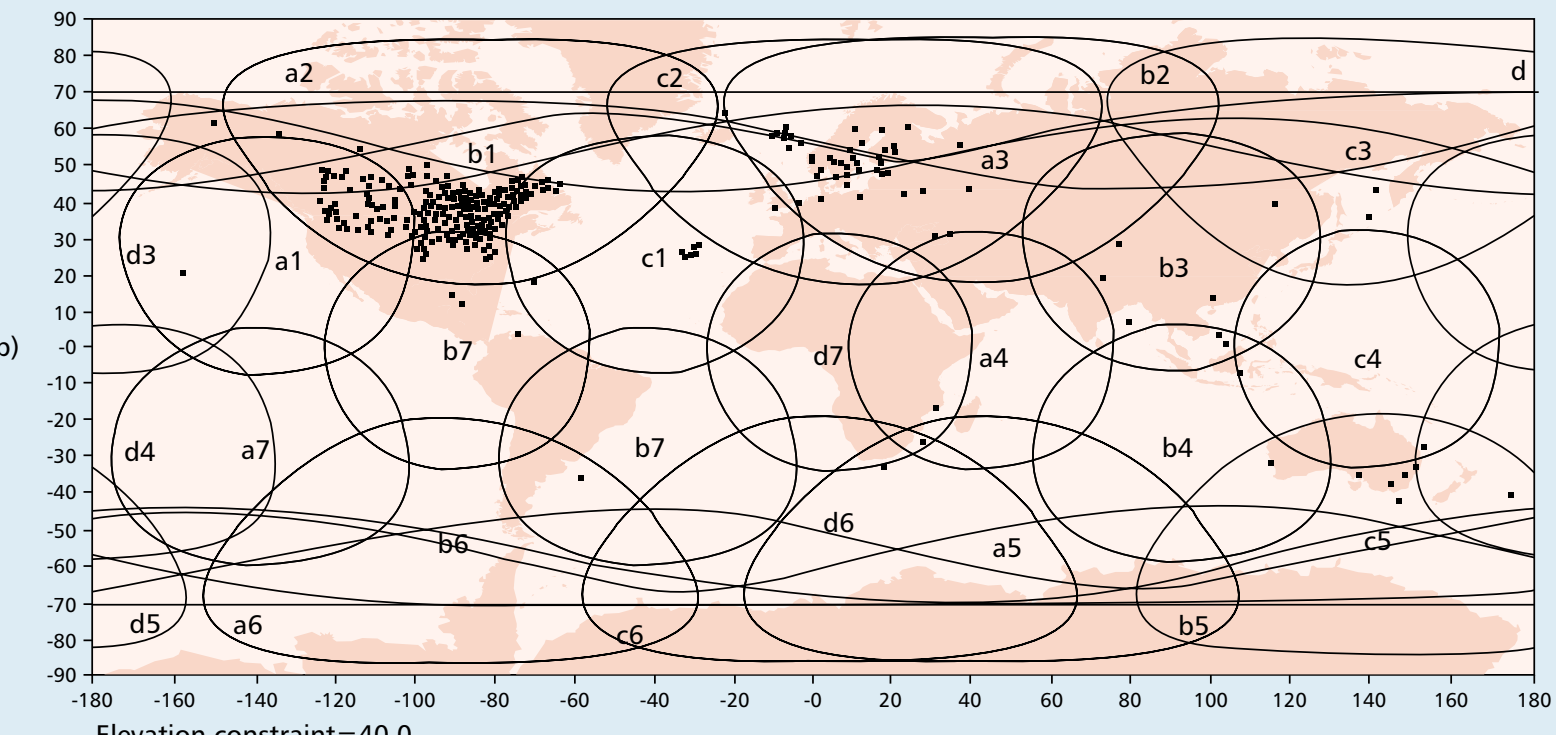

Elevation constraint $=40.0$

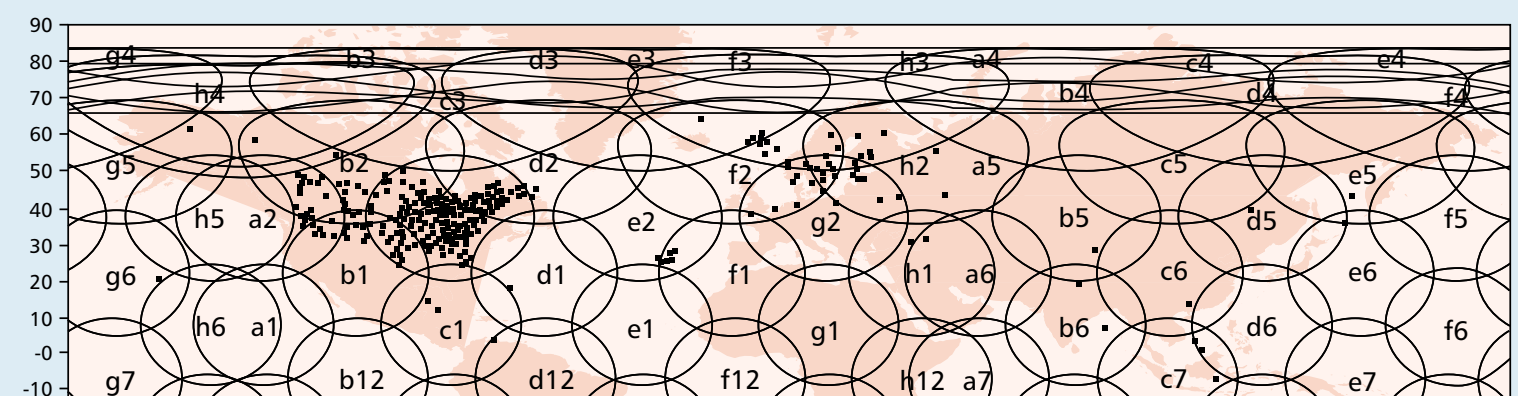

(c)

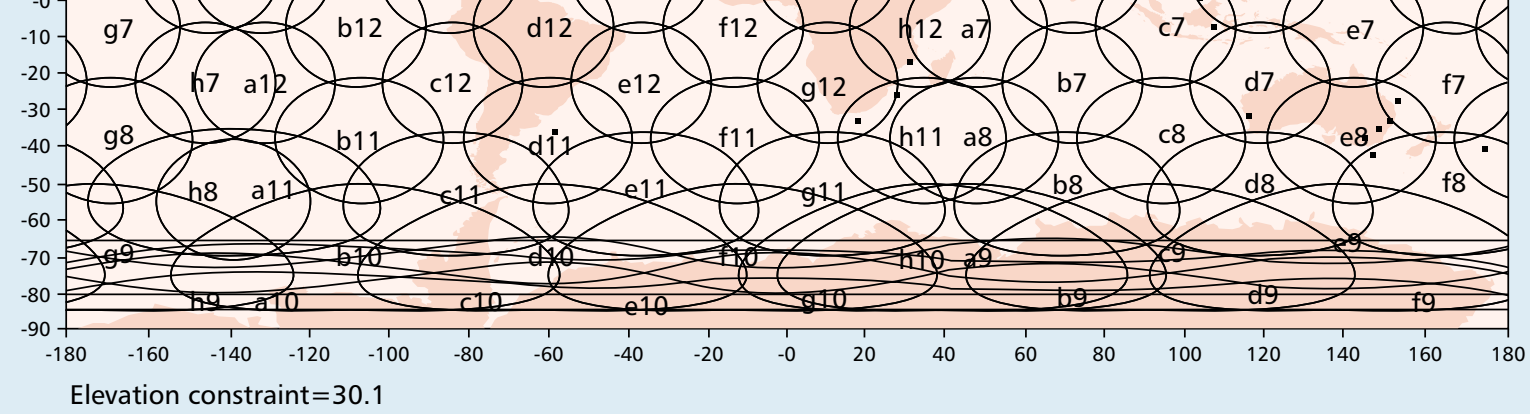

Figure 3. Coverage obtained by the selected satellite constellations: a) inclined MEO; b) polar MEO; $c$ ) polar LEO. 


\section{The signaling \\ protocols for \\ calling and \\ connection \\ control can be \\ reused (based on \\ the ITU-T Q.2931 \\ standard); \\ moreover, the \\ traffic and network \\ management \\ functions share \\ common \\ characteristics.}

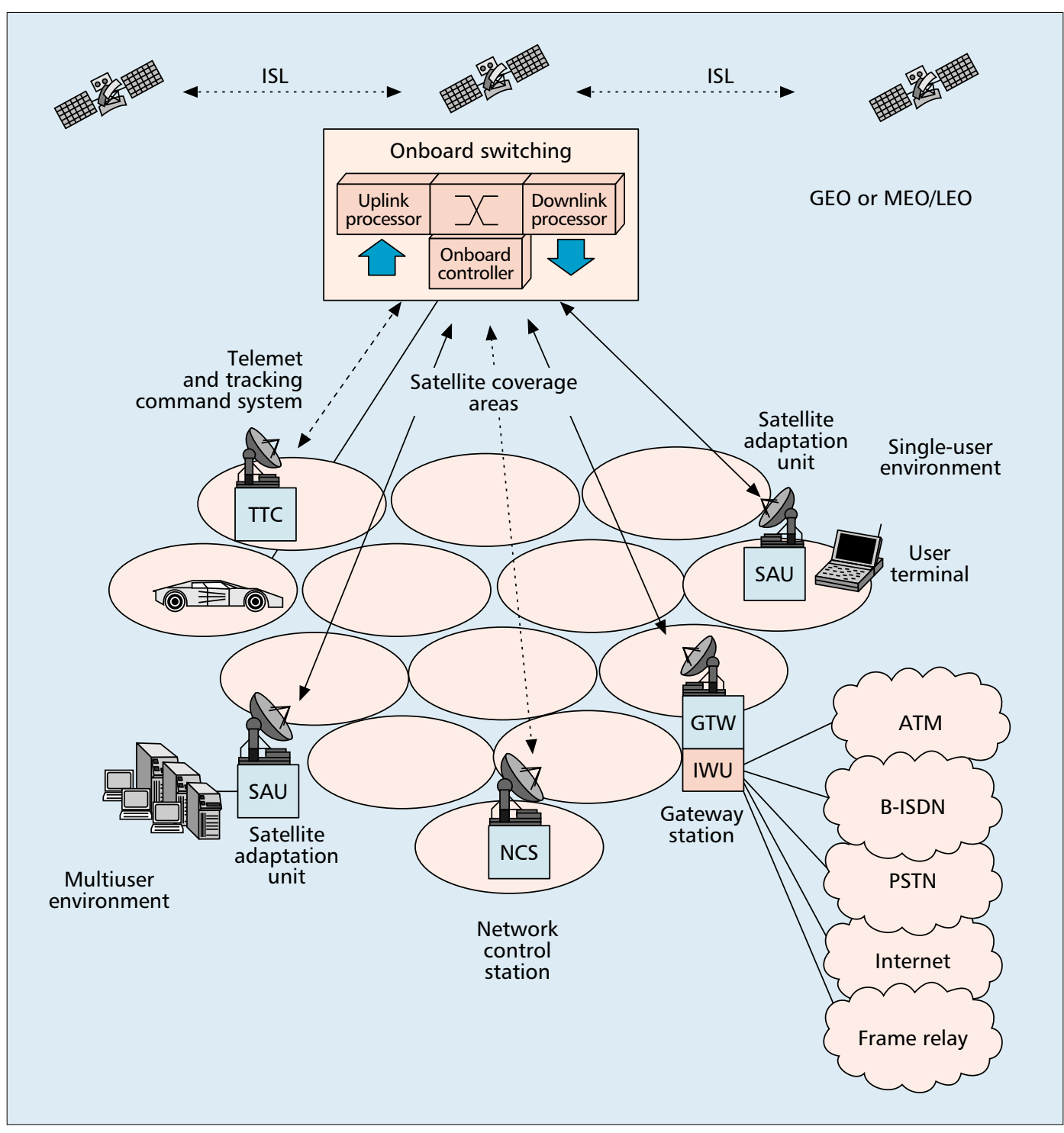

Figure 4. Dynamic multispotbeam satellite network architecture.

number of GTW stations. For example, in a global MEO system with no intersatellite links, a total number of less than 10 GTWs can provide full connectivity to the land masses most of the time. A LEO system will require tens to hundreds of GTWs, but this number can be reduced with the use of ISLs. The satellite adaptation unit (SAU) performs all the necessary user terminal protocol adaptation to the satellite protocol and can provide an access interface very similar to the standard ATM user-network interface (UNI); therefore, the required interworking at the terminal and GTW is minimized. The signaling protocols for calling and connection control can be reused, based on International Telecommunication Union - Telecommunication Standardization Sector (ITU-T) Recommendation Q.2931; moreover, the traffic and network management functions share common characteristics. The SAU also includes all the physical layer functions such as channel coding, modulation/demodulation, the radio frequency parts, and the antenna section. The network control station (NCS) is a central enti- ty used in a GEO satellite system that provides the overall control of satellite network resources, routing operations, and user database interrogation. In non-GEO systems, these operations are usually performed on different GTWs. In Fig. 4, an onboard satellite processing unit is also shown that performs multiplexing/demultiplexing, channel coding/decoding, and ATM-like switching by using a multispot beam configuration.

\section{ROUTING AND DIMENSIONING OF ISLS IN LEO SYSTEMS}

LEO systems with small delay are a very attractive approach for Internet in the sky.

Future broadband LEO systems will increasingly rely on an ISL network with time-variant topology. The ISLs among LEO satellites form a complex mesh network topology. While intraplane ISLs can be maintained permanently, interplane ISLs are switched off due to contrarotating orbits, and dynamic routing algorithms must be used to consider this time-variant 
topology. A new concept for connection-oriented ATM-based routing in periodically time-variant ISL networks has been developed [5]. This is called discrete-time dynamic virtual topology routing (DT-DVTR). DT-DVTR works completely offline (i.e., prior to system operation). In the first step, a virtual topology is set up for all successive discrete time steps of the system period (this is the least common multiple of the orbit period and the Earth's rotation period), thus providing instantaneous sets of alternative paths between all origin-destination pairs. A number of consecutive routing tables are stored onboard and retrieved when the topology changes. In the second step, path sequences over a series of time intervals are chosen according to certain optimization procedures in order to obtain the best path.

An ATM version of DT-DVTR, together with a sophisticated path sequence selection, has achieved good performance with respect to path switching offset even in an environment with switched ISLs, as encountered in polar constellations like Iridium.

For the capacity dimensioning of ISL networks, inclined Walker constellations [4] were considered by deriving a reference topology for the M-Star system. Inclined Walker constellations also allow permanently setting up a number of interplane ISLs that can be maintained with acceptable pointing, acquisition, and tracking, and this is an important feature in real-time connection-oriented services in order to avoid problems due to path switching.

Routing/splitting approaches and algorithms derived from terrestrial networks can be reused by taking into account the time variance constraints of the LEO ISL scenario.

Two appropriate target functions can be used for ISL network dimensioning:

- Minimize the worst case link (WCL) capacity, which is the maximum capacity required on any link at any time.

- Minimize the worst case node (WCN) capacity, which is the maximum capacity required on any satellite node at any time.

In [6], a comparison of basic routing/splitting approaches was presented to understand how they operate on the topology. In the M-star ISL topology, the interplane ISLs over mid-latitudes are always critical (i.e., many loaded links). The results show that the minimization of the worst case link (WCL) capacity works very well in this scenario by shifting the traffic toward the lower loaded links. Of course, the most important trade-off from the user's or QoS point of view is between the WCL load minimization target (this is mainly important for network performance and system cost) and the correlated degradation in terms of path delay encountered. For appropriate parameter settings, the increase of path delay is kept fairly low, around 5-10 percent with respect to the reference case of routing each origin-destination traffic entirely over the shortest path alone.

\section{ResouRCE MANAGEMENT}

S-UMTS - The spectrum assigned to LEO MSSs is reduced with respect to the expected market diffusion, and optimized radio resource management strategies have to be investigated.
In the fixed channel allocation (FCA) technique, a set of channels is permanently assigned to each cell (this is the spotbeam footprint on the Earth) according to the reuse distance, and therefore only a limited number of calls can be simultaneously accepted in each spotbeam. The calls are blocked when all the resources are in use.

In the dynamic channel allocation (DCA) technique, any system channel can be temporarily assigned to any cell, according to the reuse distance, and the new calls are allocated to channels that can guarantee no interference with existing calls. DCA strategies, due to their inherent capability to respond to time-variant unbalanced traffic load patterns, are suitable for use as management methods in LEO systems [7].

A modified DCA technique called simplified maximum packing (SMP) performs well under nonuniform traffic patterns, thus outperforming the other DCA methods. The use of cost functions and the possibility of channel rearrangement at the beginning/end of a call achieve a lower blocking capability by employing nominal channels and reducing the average reuse distance. The main disadvantage is high computational effort.

In LEO-MSSs, interbeam handover requests are extremely frequent during the call lifetime (one could expect that a call experiences an interbeam handover once a minute or more often), and at each beam change the call may be dropped due to an unsuccessful handover. From the user standpoint, interruption of a conversation is less desirable than blocking a new incoming call. Hence, LEO-MSSs require specific techniques that prioritize the service of handover requests with respect to new call attempts in order to reduce as much as possible the call dropping probability $[2,8]$.

When a mobile subscriber with a call in progress leaves a cell and enters an adjacent cell, there is an area (overlap area) where this mobile user receives a signal with an acceptable power level from both cells. An interbeam handover strategy based on queuing of handover requests $(\mathrm{QH})$ is essential to guarantee a suitable QoS to mobile users in LEO MSSs.

The most common queuing discipline is firstin first-out (FIFO), where handover requests are queued according to their arrival instants. In another scheme, called last useful instant (LUI), when a handover request is queued, the controller on the satellite exactly estimates the time spent by the mobile in order to cross the overlap area. A new handover request is stored in a queue position according to its residual time in the overlap area. In this way, the system tries to serve first the most urgent handover requests.

$\boldsymbol{S}-\boldsymbol{A T M}$ - An adaptive bandwidth reservation scheme (ABRS) for radio resource management in dynamic satellite networks applicable to both FCA and DCA is proposed in [9]. A metric called mobility reservation status is updated every time a new call is accepted or released, or a handoff to another spotbeam is performed. This provides information about the bandwidth requirements of all active connections in a specific spotbeam in addition to the "possible"

In the fxed
channel
allocation
technique, a set
of channels is
permanently
assigned to each
cell according to
the reuse
distance, and
therefore only a
limited number
of calls can be
simultaneously
accepted in each
spotbeam.

technique, a set

of channels is

assigned to each

cell according to

the reuse

distance, and

therefore only a

of calls can be

simultaneously

spotbeam. 
The great

advantage of the

ABRS lies in the

absence of a

fixed predefined

way of handling

calls in a wireless

network. This

incorporates a

service class

separation in

such a way that it

can be adjusted

to any multi-class

traffic scenario. bandwidth requirements of mobile terminals currently connected to the neighboring spotbeams. Two different threshold values are used for handling the new calls and handoff requests. A new call is accepted in a spotbeam if there are enough channels available for that spotbeam and the mobility reservation status of adjacent spot-beams has not exceeded a predetermined threshold. If there are channels available on the target spotbeam and the mobility reservation status of that beam is less than another threshold, handoffs from one spotbeam to another are successful. The bandwidth reservation mechanism is simple enough to be executed in real time and also provides the flexibility to map, in a dynamic way, the service requirements into the non-GEO network performance features. In addition, due to the dynamic bandwidth reservation, each service class maintains a QoS profile that was accepted or negotiated during the call setup phase and can easily be modified within the call. The great advantage of the ABRS lies in the absence of a fixed predefined way of handling calls in a wireless network. This incorporates a service class separation in such a way that it can be adjusted to any multiclass traffic scenario.

\section{Mobility Management}

S-UMTS - In satellite personal communication networks, FES in the ground network could have the same functionality as that of the mobile switching center (MSC) in the GSM network with one user's location area only. On receiving a user-terminated call, it is preferable to route the call to an FES with high probability of maintaining the call throughout its duration, since inter-FES handovers can involve international rerouting of the call with an associated delay and possibly different call charge rates if the new FES is provided by a different network operator. As satellites move in and out of view, a mobile user should attempt to use the satellite that is providing the most optimal channel, even if this satellite is not directly connected with the FES currently handling the call. To restrict the user's choice of satellites to those only offering connectivity with the current FES could compromise the QoS (which is directly related to the signal quality between the user terminal and satellite). Due to nonGEO satellites' motion with respect to the earth's surface, the area around the FES where connectivity is currently provided is continuously changing in time. Consequently, the optimum satellite connection area is the area around the Earth station where the satellite with the highest elevation angle (the most likely to offer good channel conditions) to the mobile terminal will provide connectivity to the FES.

By considering inter-FES handover probabilities for FESs at various latitudes for a typical mobile call length distribution, at lower probabilities the second highest satellite provides connectivity with an FES over a larger area than the highest satellite.

A scenario of mobility management schemes is considered where there exist in-call FES handover agreements between operators. Due to the dynamic motion of satellites in a non-GEO con- stellation, the adoption of the GSM approach to mobility management results in a large increase in associated signaling. In order to overcome this problem, an alternative method of calculating (at location update time) the probability that a future user-terminated call requires FES handover was illustrated in [10] for a given location area radius.

In this mobility management scheme, mobile terminals make a location update after moving a predetermined distance from their last point of contact with the network. The user's movement is monitored by a satellite-based positioning system. The size of the area around the FES where a terminal can make a location update with a probability of no future in-call FES handover being required above a certain threshold value varies with the location area radius. The results show that the second highest satellite can have a large impact on the FES service area, whereby the QoS specified by the service providers in terms of inter-FES handover probability or call dropping due to loss of FES connectivity determines the area size.

\section{S-ATM}

Many mobility issues related to wireless ATM networks, such as the virtual connection tree concept, can be applied to dynamic satellite ATM networks [9]. According to the original virtual connection tree algorithm, mobile terminals can freely roam around a large area covered by several radio access points and execute handoffs by simply using a predefined set of virtual circuit numbers for any particular activated path. A mobile user is admitted to a virtual connection tree during the call establishment phase, and lookup tables are created at intermediate switching points of the connection tree.

In an S-ATM network, the root of a connection tree can be either a GTW station that serves a particular coverage area or an ATM switch of the fixed network. The leaves will be the input parts corresponding to a single or group of spotbeam(s) that feed the onboard switch. The virtual trees will be dynamically established and released according to the satellite movements. Every time a mobile station accesses the satellite station in order to initiate a call, its exact position can be calculated, and the next handover time predicted with high accuracy. The user handoff times and direction with respect to the moving multispotbeam pattern can be predicted during the call initialization phase. This gives a great advantage over terrestrial mobile systems, since the list of the visited spotbeams can be predefined.

The satellite movement being predicted, a new approach was proposed in [9] to balance the system call blocking probability with the call dropping probability due to unsuccessful handoffs for different types of services.

\section{PRotocols}

S-ATM - Two main scenarios can be applied to the broadband satellite networks to accommodate ATM-compatible equipment $[2,4]$ :

- ATM protocol encapsulation and use of fast packet switching on the satellite segment for user establishment and management. Easy to implement, the satellite protocol is 
designed to transparently support various protocol standard due to satellite-specific interfaces and gateways. No modifications to existing protocol standards are needed, but packet overheads increase, resulting in protocol inefficiencies.

- A highly integrated solution with the ATM protocol stack; a specific satellite-oriented ATM protocol layer where an S-ATM layer replaces the standard ATM layer with all the required modifications on the cell header and its function; a medium access control (MAC) using MF-TDMA or CDMA and the radio physical layer reside below the S-ATM.

There are some strong similarities between the two protocol scenarios, included in the term ATM compatibility. This term implies the existence of a common fixed size information unit that traverses all the different network interfaces and can carry both control and user data. This relies on a very fast switching fabric at the lower layers of the communication protocols. The higher-layer protocols in different planes (i.e., control and user planes) establish, maintain, release, or transfer user data during a network connection. It is expected that in the next two to five years most Ka-band proposals will adopt (for their satellite network infrastructure) a new modified version of the ATM protocol layer (SATM), which will be satellite-specific with no major modifications to the ATM protocol stack. The S-ATM cell header fields will carry essential routing and control information for the satellite segment, and different techniques such as partial packet discard (PPD) for reliable non-real-time data transfer could be used to detect erroneous cells at the satellite switch [4].

PRMA - The classical PRMA scheme has been proposed for terrestrial microcellular systems. This is based on slotted $A L O H A$ access and TDMA transmission mode, and combines random access with a slot reservation scheme [11], thus exploiting silent phases of voice sources to multiplex more conversations on the same channel. Therefore, the assignment of time slots to terminals is not fixed, but dynamically handled on the basis of the presently active terminals. When selecting optimum values for both permission probabilities and frame duration, PRMA is advantageous with respect to TDMA in managing integrated voice and data traffic, and provides the same capacity improvements exhibited in terrestrial microcellular networks.

By considering a real-time variable bit rate (rtVBR) service for voice and an available bit rate (ABR) service for data, a modified PRMA scheme, PRMA-HS with hindering states, where a terminal does not stop contending while it waits for receiving the outcome of a previous reservation attempt, provides high efficiency and is quite insensitive to the variations of round-trip delay in LEO systems. Therefore, PRMA-HS can be envisaged as a good solution for a unified MAC protocol for the terrestrial and satellite components of future mobile communication systems.

An advanced PRMA (A-PRMA), where transmissions do not depend on permission probability, is also analyzed for rt-VBR traffic. The results, evaluated according to the cell loss ratio, show that this technique is appropriate for transmitting integrated voice and data even if this cannot be fully suitable for guaranteeing all the QoS parameters.

\section{AIR INTERFACE ISSUES}

The propagation characteristics and satellite diversity are two important issues, since future mobile and personal satellite/terrestrial services could adopt a non-GEO satellite constellation. Propagation measurements at the L-band were previously performed by several organizations, for LEO, MEO, HEO, and GEO systems [12, 13]. At higher frequencies (EHF band), a new measurement campaign shows that the signal on the direct path is subject to shadowing, and echoes appear very rarely in rural roads. Compared to the results at the L-band obtained at the same location, the number of echoes is much smaller and echo attenuation higher at the EHF band. In urban environments, the shadowing for the direct signal is stronger, while the number of echoes is higher.

For the EHF band a channel model is derived by Lutz from the measurement campaign [2, 13]. The channel behavior is characterized by two states: a good channel state with Ricean distribution and a bad channel state with Rayleigh distribution, corresponding to the unshadowed and shadowed channels.

Given the power limitation, especially in the uplink, the solution to reduce such shadowing effects is path diversity or satellite diversity. Satellite diversity is the general case where at least two satellites cover a given location on Earth. This results in an overlap of satellite footprints that define the area where the satellites are viewed above a specified elevation angle, and is a common feature pg all non-GEO constellations providing continuous coverage of the service area.

In [14] the single-satellite statistical model was extended to study the simultaneous behavior of two partially correlated links by introducing the shadowing correlation coefficient.

In [15] the quantification of the correlation coefficient for different types of environments based on physical models is presented for extending the single link study to a multilink scenario.

The impact of satellite diversity on the capacity of non-GEO satellite systems for both fixed and dynamic coverage was outlined in [14]. Satellite antennas can be configured to cover the service area with either fixed beams or beams dynamically varying in shape and size by using active antenna arrays. In both cases, continuous coverage of the service area is considered the most important requirement.

Dynamic coverage allows a significant increase in system capacity with high satellite diversity probability ( $>90$ percent); therefore, this is an appealing feature for future system design.

\section{CDMA SYSTEMS}

The 3G system (UMTS) adopts W-CDMA for its full frequency reuse, easy adaptation to variable rate services, and suitability to adaptive interference mitigation techniques. The CDMA technique is also the foundation of S-

\author{
The propagation \\ characteristics \\ and satellite \\ diversity are two \\ important issues, \\ since future \\ mobile and \\ personal \\ satellite/terrestrial \\ services could \\ adopt a non-GEO \\ satellite \\ constellation
}




UMTS adopts
W-CDMA for its
full frequency
reuse, easy
adaptation to
variable rate
services, and
suitability
to adaptive
interference
mitigation
techniques. The
CDMA technique
is also the
foundation of
S-UMTS.

UMTS. Codes and new receiver schemes and concepts suitable for CDMA systems have been analyzed [2].

\section{TCH CODES}

Tomlinson, Cercas, Hughes (TCH) codes are a class of binary, nonlinear, nonsystematic, and cyclic block codes, with length $n=2^{m}$ being any positive integer. These exhibit good performance for forward error correction (FEC) applications and undertake maximum likelihood soft decision decoding with a very simple decoder structure by using digital signal processing (DSP) techniques.

Further analysis of the correlation properties of TCH sequences (i.e., TCH codewords taken as sequences) indicated that it is possible to identify some sets of sequences that exhibit good auto- and cross-correlation. These are very important properties since the performance of CDMA systems depends not only on the cross-correlation properties of the sequences in order to minimize interuser interference, but also on their autocorrelation due to the synchronization process. Therefore, TCH codes can also be employed for spreading by taking advantage of their simplified correlation receiver to detect different users in a CDMA environment.

\section{THE CDMA RECEIVER}

An important CDMA feature is the possibility to use a time diversity structure joint with multiuser detection (MUD) to oppose multipath fading effects. The conventional single-user receiver for CDMA communications does not exhibit acceptable performance due to multiple access interference (MAI).

Although optimum multiuser algorithms offer huge potential capacity and significantly improved performance, these are too complex to be implemented. Suboptimal solutions like decorrelating detectors or multistage receivers were investigated. A multiuser cancellation detection scheme for SW-CDMA communication systems is considered.The receiver has a single-stage architecture in which the parallel interference cancellation (PIC) of all interfering users with respect to the user of interest is performed on a selective basis (S-PIC) before the final symbol decision. The basic assumption of the proposed detector is to divide matched filter outputs into two different groups according to the power level of received signals. The reliable signals are directly detected and cancelled in the whole received signal before making the decision on unreliable signals or replicas, without any further processing delay.

Therefore, the proposed interference cancellation approach achieves better performance with respect to the Rake receiver while keeping lower complexity.

Different schemes of blind adaptive multiuser detectors for DS/CDMA satellite communication systems with binary phase shift keying (BPSK) modulation are analyzed and their performance evaluated for LEO/MEO mobile satellite systems. The proposed receivers are used at the end of the uplink satellite channel in the base station, so the communication system is characterized by a lack of synchronism between the users in multipath fading satellite channels. These schemes are based on the blind adaptive multiuser detector proposed by Verdù, Honig, and Madhow: in the former scheme, a scalar blind receiver consists of different detectors (one for each replica), while in the latter a vectorial detector considers the received signal as a whole, with a better complexity-performance trade-off. The proposed multiuser detectors exhibit remarkable near-far resistance and require the knowledge of the same parameters as those needed by the conventional single-user receiver (i.e., the desired user's signature waveform and its timing), while no training sequence is needed, only essential information about all the users (i.e., the number of active users and the processing gain) [16].

\section{CONCLUSIONS}

It is internationally recognized (UMTS/IMT2000) that satellite systems are needed to provide the required global coverage of future mobile and personal communications. COST252 for analyzing the new generation of satellite personal communications with high data-rate services is therefore completely accounted for.

According to the scenarios (S-UMTS, SATM, S-IP scenarios) identified for future mobile satellite networks, the main innovative objectives of COST252 are in the following fields: a scheduling entity for the MF-TDMA MAC protocol with guaranteed levels of QoS; routing algorithms (DT-DVTR) and dimensioning of intersatellite links in LEO satellite systems; a resource management area analyzing the DCA technique in GEO and LEO constellations; and protocols examining the PRMA protocol in detail. Important results are also achieved in the CDMA environment for TCH codes and new DS-CDMA receivers. For specific research activities and results of COST252, the reader is referred to its Final Report [2].

Another important issue for next-generation mobile and fixed satellite services is the use of IP over satellite, which is one of the main topics to be addressed by future research.

\section{ACKNOWLEDGMENTS}

The authors would like to thank the COST252 participants whose contributions and enthusiastic participation led to the success of the COST252 project, and the development of the concepts and views presented in this article.

\section{PARTICIPATING INSTITUTIONS}

$\begin{array}{lr}\text { Bradford University } & \text { UK } \\ \text { CSELT } & \text { I } \\ \text { Corporate Technology Swiss Telecom } & \text { CH } \\ \text { DLR } & \text { D } \\ \text { Ecole Polytechnic. Federal de Lausanne } & \text { CH } \\ \text { Ecole Superior Telecom } & \mathrm{F} \\ \text { France Telcom -CNET } & \mathrm{F} \\ \text { Institute Superior Tecnico } & \mathrm{P} \\ \text { Aveiro Inst. Telecom. } & \mathrm{P} \\ \text { Institute Joseph Stefan } & \mathrm{SL} \\ \text { University of Surrey } & \mathrm{UK}\end{array}$


University Rome Tor Vergata

University Catholic Louvain

University of Florence

Warsaw University of Technology

Aristotle University of Thessaloniki

University of Athens

Deuthsche Telecom

SINTEF

\section{Web Site}

The COST252 Web site is at http://lenst.det. unifi.it/cost 252

\section{REFERENCES}

[1] R. E. Sheriff, "Current Activities in COST252/WG 1: Strategic Scenarios and Feasibility Studies," COST252 TD, vol. 98, no. 11 .

[2] E. Del Re and L. Pierucci, "Satellite Personal Communications for Future-Ggeneration Systems," Final Report: Cost252 Action, Springer, 2002.

[3] M. Mohorcic and R. E. Sheriff, "Non-Geostationary Satellite Constellations: Visibility Aspects," COST252 TD, vol. 98 , no. 21.

[4] I. Mertzanis et al., "Protocol Architectures for SatelliteATM Broadband Networks," IEEE Commun. Mag., vol. 37, no. 3, Mar. 1999, pp 46-54.

[5] M. Werner, "A Dynamic Routing Concept for ATMBased Satellite Personal Communication Networks," IEEE JSAC, vol. 15, no. 8, Oct. 1997, pp. 1636-48.

[6] F. Wauquiez and M. Werner, "Capacity Dimensioning of Intersatellite Link Networks in Broadband Leo Satellite Systems," COST252 TD, vol. 98, no. 24, Sept. 1998

[7] E. Del Re, R. Fantacci, and G. Giambene, " Handover Queuing Strategies with Dynamic and Fixed Channel Allocation Techniques in Low Earth Orbit Mobile Satellite Systems," IEEE Trans. Commun., vol. 47, no. 1, Jan. 1999, pp. 89-102.

[8] E. Del Re, R. Fantacci, and G. Giambene, "Different Queuing Policies for Handover Requests in Low Earth Orbit Mobile Satellite Systems," IEEE Trans. Vehic. Tech., vol. 48, no. 2, Mar. 1999, pp. 448-58.

[9] I. Mertzanis, R. Tafazolli, and B.G Evans, "A New Approach for Radio Resource Management in Multimedia Dynamic Satellite Networks," 17th AIAA Int'I. Commun. Sat. Sys. Conf. \& Exhibit, Yokohama, Japan, 23-27 Feb. 1998.

[10] T. Sammut, "Mobility Management Related Signaling for a MAGSS-14 Based Satellite Personal Communications Network (S-PCN)," COST227D, NTUA, Athens, Greece, 1994.

[11] Del Re, R. Fantacci, G. Giambene, and S. Walter "Performance Analysis of an Improved PRMA Protocol for Low Earth Orbit Mobile Satellite Systems," IEEE Trans. Vehic. Tech., vol. 48, no. 3, May 1999, pp. 985-1001.

[12] E. Lutz et al., "The Land Mobile Satellite Communication Channel -Recording, Statistics and Channel Model," IEEE Trans. Vehic. Tech., vol. 40, 1991, pp. 375-86.

[13] G. Butt, B. G. Evans, and M. Richharia, "Narrowband Channel Statistics from Multiband Propagation Measurements Applicable to High Elevation Angle LandMobile Satellite Systems," IEEE JSAC, vol. 10, 1992, pp. 1219-26.

[14] H. Bischl, A. Jahn, and E. Lutz, "Wideband Channel Model for UMTS Satellite Communications," TD vol. 98, 05 temp. doc. inside COST252.
[15] C. Tzaras, B. G. Evans, and R. S. Saunders, "A PhysicalStatistical Analysis of the Land Mobile Satellite Channel," IEE Elect. Lett. vol. 34, no. 13, 1998, pp. 1355-57.

[16] E. Del Re et al., "A Low-complexity Multiuserr Detector for up-link CDMA Mobile Radio Communication," TD vol. 98, 10 temp. doc. inside COST252.

\section{BIOGRAPHIES}

ENRICO DEL RE [SM] (delre@lenst.det.unifi.it) received a Dr. Ing. degree in electronics engineering from the University of Pisa, Italy, in 1971. Until 1975 he was engaged in public administration and private firms, involved in the analysis and design of telecommunication and air traffic control equipment and space systems. Since 1975 he has been with the Department of Electronics Engineering of the University of Florence, Italy, first as a research assistant, then as an associate professor, and since 1986 as a professor. During the academic year 1987-1988 he was on leave from the University of Florence for a nine-month period of research at the European Space Research and Technology Centre of the European Space Agency, The Netherlands. His main research interests are digital signal processing, mobile and satellite communications, and communication networks, on which he has published more than 150 papers in international journals and conferences. $\mathrm{He}$ is coeditor of the book Satellite Integrated Communications Networks (North-Holland, 1988), and one of the authors of the book Data Compression and Error Control Techniques with Applications (Academic, 1985). He has been chairman of European Project COST 227, "Integrated Space/Terrestrial Mobile Networks," and EU COST Action 252, "Evolution of Satellite Personal Communications from Second to Future Generation Systems." He received the 1988-1989 premium from the IEE (UK) for the paper "Multicarrier Demodulator for Digital Satellite Communication Systems." He is head of the Digital Signal Processing and Telematics Laboratory of the Department of Electronics and Telecommunications of the University of Florence. He is a member of the Executive Board of the Italian Interuniversity Consortium for Telecommunications (CNIT). He is a member of the European Association for Signal Processing (EURASIP).

LAURA PIERUCCI (pierucci@lenst.det.unifi.it) received her Dr. Eng. degree in electronics engineering from the University of Florence, Italy, in 1987. Until 1991 she was a research consultant for private firms in the fields of ultrasonic applications in medicine and the development of radio mobile cellular systems. In 1989 she was winner of a fellowship of the National Research Council (CNR.) on "Study of Devices in Optical Fibre and of Optical Multiplexing Techniques to Use in Data Transmission Network." Currently, she is with the Department of Electronics and Telecommunications of the University of Florence, as assistant professor in the fields of telecommunications and digital signal processing. Her main research interests are in digital signal processing, adaptive systems, neural network, antenna array, MIMO systems, and the general area of mobile and satellite communication systems. Since 1990, she took part in different national projects of MURST and the Italian Space Agency (ASI) and was involved in the European Projects ISIS Interactive Satellite multimedia Information System (1995-1998), Galenos - Generic Advanced Low Cost trans European Network Over Satellite (1999-2001), Hermes (1998-2002) on the fields of telecommunications systems on terrestrial and satellite networks and their applications such as tele-education and tele-medicine. She was the scientific secretariat of EU COST Action 252, "Evolution of Satellite Personal Communications from Second to Future Generation Systems" (1996-2000).
Satellite systems

are needed to

provide the

required global

coverage of

future mobile

and personal

communications.

COST252 Action

for analyzing the

new generation

of Satellite

Personal

Communications

with high

data-rate services

is therefore

completely

accounted for. 